\title{
Microscopic Rates of Peptide-Phospholipid Bilayer Interactions from Single-Molecule Residence Times
}

Grant A. Myers, Daniel A. Gacek, Eric M. Peterson, Christopher B. Fox, and Joel M. Harris

Departments of Chemistry and Bioengineering, University of Utah, 315 South 1400 East, Salt Lake City, Utah 84112

\section{Supporting Information}

Table of Contents:

Page Number

Analysis of the Differential Equations for Single-Molecule Binding

MALDI data for GLP1 labeled with Cy3B

Optimization of Imaging Algorithm to Bridge Photoblinking Events

S-6

Intensity Distribution of Cy3B-labeled Fluorescence Spots

S-7

Isotherms Comparing Binding of Cy3B-labeled GLP-1 to DPPC with Control Samples

Cumulative Residence-Time Histogram Plotted on a Log-Linear Scale

Residence-time Histogram Kinetics versus Laser Power

Comparison of GLP-1 Kinetic Parameters Measured on Different Systems 


\section{Analysis of the Differential Equations for Single-Molecule Binding.}

1. General system of differential equations.

The system of differential equations associated with three-state consecutive reversible first-order kinetics includes three expressions for the rate of change of the number of molecules in each of the three states as shown here in linear algebra form, given as the dot product of the microscopic rate coefficient matrix with a vector of the instantaneous concentrations (volume and surface) of the three populations:

$$
\left(\begin{array}{c}
\partial F / \partial t \\
\partial W / \partial t \\
\partial S / \partial t
\end{array}\right)=\left(\begin{array}{ccc}
-k_{1} \lambda^{-1} & k_{2} \lambda^{-1} & 0 \\
k_{1} & -k_{2}-k_{3} & k_{4} \\
0 & k_{3} & -k_{4}
\end{array}\right) \bullet\left(\begin{array}{c}
F(t) \\
W(t) \\
S(t)
\end{array}\right)
$$

where, $k_{l}$ is the heterogeneous rate constant for molecules binding to the surface from the solution, $k_{2}$ is the rate constant for molecules unbinding from the surface, $\lambda^{-1}$ is the ratio of the surface area of the bilayer to the volume of the system that accounts for the change in dimensionality for going from volume of solution to the surface area of bilayer, $k_{3}$ is the rate constant for molecules changing from the weakly bound to the strongly bound state. For example, the rate of change of the number of molecules in the $S$ state, $\partial S / \partial t$, is given by $k_{3} W(t)-k_{4} S(t)$. The solutions to this system of differential equations for the functions representing the time dependence of the number of molecules of each of the components have the following general form: $X(t)=X_{e q}+P_{1 X} e^{-r_{1} t}+P_{2 X} e^{-r_{2} t}$, where the equilibrium and pre-exponential coefficient terms are complicated functions of the microscopic rate constants, $k_{l}$ through $k_{4}$, and are unique for each component. In contrast, the rate terms, $r_{l}$ and $r_{2}$, are the same for all components and are given ${ }^{1}$ by the following equations:

$$
\begin{aligned}
& r_{1}=\frac{1}{2}\left[k_{1} \lambda^{-1}+k_{2}+k_{3}+k_{4}+\sqrt{\left(k_{1} \lambda^{-1}+k_{2}+k_{3}+k_{4}\right)^{2}-4\left(k_{1} k_{3} \lambda^{-1}+k_{1} k_{4} \lambda^{-1}+k_{2} k_{4}\right.}\right] \\
& r_{2}=\frac{1}{2}\left[k_{1} \lambda^{-1}+k_{2}+k_{3}+k_{4}-\sqrt{\left(k_{1} \lambda^{-1}+k_{2}+k_{3}+k_{4}\right)^{2}-4\left(k_{1} k_{3} \lambda^{-1}+k_{1} k_{4} \lambda^{-1}+k_{2} k_{4}\right.}\right]
\end{aligned}
$$

Note that, as written, $r_{l}$ will always be larger than $r_{2}$. These rate expressions can be recognized as the eigenvalues ${ }^{2}$ of the coefficient matrix from Equation S1, or as roots ${ }^{3}$ of the quadratic equation: $y=x^{2}-\left(k_{1} \lambda^{-1}+k_{2}+k_{3}+k_{4}\right) x+\left(k_{1} k_{3} \lambda^{-1}+k_{1} k_{4} \lambda^{-1}+k_{2} k_{4}\right)$ Interestingly, the sum of $r_{1}$ and $r_{2}$ is equal to the sum of the microscopic rate constants, $k_{1} \lambda^{-1}+k_{2}+k_{3}+k_{4}$. The difference between $r_{1}$ and $r_{2}$ is equal to the radical term in either of the rate expressions, and the product of $r_{1}$ and $r_{2}$ is equal to $\left(k_{1} k_{3} \lambda^{-1}+k_{1} k_{4} \lambda^{-1}+k_{2} k_{4}\right)$.

The pre-exponential coefficients corresponding to the two rates, $r_{1}$ and $r_{2}$, for each of the three components are given as follows:

$P_{1 F}$
$=\frac{-k_{1} \lambda^{-1}\left(-k_{2} k_{3}-\left(k_{3}+k_{4}\right)\left(k_{3}+k_{4}+r_{1}-r_{2}\right)+r_{1} r_{2}\right) F(0)+k_{2}\left(k_{1} k_{3} \lambda^{-1}-k_{4}\left(k_{3}+k_{4}+r_{1}-r_{2}\right)+r_{1} r_{2}\right) W(0)-2 r_{1} k_{2} k_{4} S(0)}{2 r_{1} r_{2}\left(r_{1}-r_{2}\right)}$

$P_{2 F}$

$=\frac{k_{1} \lambda^{-1}\left(-k_{2} k_{3}-\left(k_{3}+k_{4}\right)\left(k_{3}+k_{4}-r_{1}+r_{2}\right)+r_{1} r_{2}\right) F(0)-k_{2}\left(k_{1} k_{3} \lambda^{-1}-k_{4}\left(k_{3}+k_{4}-r_{1}+r_{2}\right)+r_{1} r_{2}\right) W(0)+2 r_{2} k_{2} k_{4} S(0)}{2 r_{1} r_{2}\left(r_{1}-r_{2}\right)}$

(S3b)

$P_{1 W}=\frac{\left(\begin{array}{c}-k_{1} \lambda^{-1}\left(k_{1} k_{3} \lambda^{-1}-k_{4}\left(k_{3}+k_{4}-r_{1}+r_{2}\right)+r_{1} r_{2}\right) F(0) \\ -\left(k_{1}^{2} k_{3} \lambda^{-2}-k_{1} \lambda^{-1}\left(k_{3}^{2}+\left(1+k_{2}\right) k_{4}+k_{3}\left(1+k_{2}+k_{4}+r_{1}-r_{2}\right)\right)-k_{2} k_{4}\left(1+k_{2}+k_{3}-k_{4}+r_{1}-r_{2}\right)+r_{1} r_{2}\right) W(0) \\ +k_{4}\left(k_{1}^{2} \lambda^{-2}-3 k_{2} k_{4}+k_{1} \lambda^{-1}\left(k_{2}-2 k_{3}-2 k_{4}-r_{1}+r_{2}\right)\right) S(0)\end{array}\right)}{2 r_{1} r_{2}\left(r_{1}-r_{2}\right)}$ 
$P_{2 W}=\frac{\left(\begin{array}{c}k_{1} \lambda^{-1}\left(k_{1} k_{3}-k_{4}\left(k_{3}+k_{4}+r_{1}-r_{2}\right)+r_{1} r_{2}\right) F(0) \\ +\left(k_{1}^{2} k_{3} \lambda^{-2}-k_{1} \lambda^{-1}\left(k_{3}^{2}+\left(1+k_{2}\right) k_{4}+k_{3}\left(1+k_{2}+k_{4}-r_{1}+r_{2}\right)\right)-k_{2} k_{4}\left(1+k_{2}+k_{3}-k_{4}-r_{1}+r_{2}\right)+r_{1} r_{2}\right) W(0) \\ -2 k_{4}\left(k_{1}^{2} \lambda^{-2}-3 k_{2} k_{4}+k_{1}\left(k_{2}-2 k_{3}-2 k_{4}-r_{1}+r_{2}\right)\right) S(0)\end{array}\right)}{2 r_{1} r_{2}\left(r_{1}-r_{2}\right)}$

(S3d)

$P_{1 S}=\frac{\left(\begin{array}{c}-2 k_{1} k_{3} r_{1} F(0) \lambda^{-1}-k_{3}\left(k_{1}^{2} \lambda^{-2}-k_{2} k_{4}+r_{1}+r_{2}+k_{1} \lambda^{-1}\left(k_{2}+r_{1}-r_{2}\right)\right) W(0) \\ +k_{4}\left(k_{1}^{2} \lambda^{-2}+r_{1}+r_{2}+k_{1} \lambda^{-1}\left(2 k_{2}+r_{1}-r_{2}\right)+k_{2}\left(k_{2}+k_{3}+r_{1}-r_{2}\right)\right) S(0)\end{array}\right)}{2 r_{1} r_{2}\left(r_{1}-r_{2}\right)}$

(S3e)

$P_{2 S}=\frac{\left(\begin{array}{l}2 k_{1} k_{3} r_{2} F(0) \lambda^{-1}+k_{3}\left(k_{1}^{2} \lambda^{-2}-k_{2} k_{4}+r_{1}+r_{2}+k_{1} \lambda^{-1}\left(k_{2}-r_{1}+r_{2}\right)\right) W(0) \\ -k_{4}\left(k_{1}^{2} \lambda^{-2}+r_{1}+r_{2}+k_{1} \lambda^{-1}\left(2 k_{2}-r_{1}+r_{2}\right)+k_{2}\left(k_{2}+k_{3}-r_{1}+r_{2}\right)\right) S(0)\end{array}\right)}{2 r_{1} r_{2}\left(r_{1}-r_{2}\right)}$

(S3f)

where $F(0), W(0)$, and $S(0)$ are the initial solution and surface concentrations in their respective states. At sufficiently long times $\left(\mathrm{t} \gg 1 / r_{2}\right)$, the relative concentrations of each of the three components evolve from their initial concentrations to their equilibrium values, which are given by the following equations, ${ }^{1}$ normalized to the total number of molecules in the system:

$$
\begin{gathered}
F_{e q}=\frac{k_{2} k_{4}}{k_{2} k_{4}+k_{1} k_{3} \lambda^{-1}+k_{1} k_{4} \lambda^{-1}} \\
W_{e q}=\frac{k_{1} k_{4}}{k_{2} k_{4}+k_{1} k_{3} \lambda^{-1}+k_{1} k_{4} \lambda^{-1}} \\
S_{e q}=\frac{k_{1} k_{3}}{k_{2} k_{4}+k_{1} k_{3} \lambda^{-1}+k_{1} k_{4} \lambda^{-1}}
\end{gathered}
$$

The equilibrium expressions in Equations S4 through S6 are useful for interpreting isotherm experiments, where the interfacial concentration of molecules is measured versus the solution concentration. The sum, $W_{e q}+S_{e q}$, is the total population density on the surface. The slope of the measured isotherm is the equilibrium constant for binding, $K_{0}$, which is the ratio of the total surface density of molecules, $W_{e q}+S_{e q}$, to the concentration in solution, $F_{e q}$ :

$$
K_{0}=\frac{W_{e q}+S_{e q}}{F_{e q}}=\frac{k_{1} k_{4}+k_{1} k_{3}}{k_{2} k_{4}}
$$

Solving this equation for $k_{l}$ :

$$
k_{1}=K_{0} \frac{k_{2} k_{4}}{k_{3}+k_{4}}
$$

Allows the adsorption rate, $k_{1}$, to be determined from the slope of the isotherm and the values of $k_{2}, k_{3}$, and $k_{4}$ obtained from single-molecule histograms as described in the following section.

2. Analysis of single-molecule residence-time histograms.

Two kinetic constraints are present in the histograms of residence times of molecular visits to the interface. First, by aligning the observed events to a common origin at $t=0$, an initial condition arises for the normalized population where $W(0)=1$ and $S(0)=0$. A second constraint derives from the low surface densities interrogated in these experiments so that probability of double-occupancy within an interrogated spot area, $A$, is small and, thus the adsorption rate can be neglected in the histogram analysis so that $k_{1}=0$. This is an equivalent constraint as having the rate of a molecule binding within an interrogated spot area, $k_{1} A$ [GLP], be slower than the average off-rate, $k_{2} k_{4} /\left(k_{3}+k_{4}\right)$; see the results in Section 4.1 in the main body of the manuscript. 
These constraints greatly simplify Equations $\mathrm{S} 2$ and $\mathrm{S} 3$. When $k_{l}$ and $S(0)$ are zero, the rate terms $\mathrm{r}_{1}$ and $\mathrm{r}_{2}$ are given by:

$$
\begin{aligned}
& r_{1}=\frac{1}{2}\left[k_{2}+k_{3}+k_{4}+\sqrt{\left(k_{2}+k_{3}+k_{4}\right)^{2}-4 k_{2} k_{4}}\right] \\
& r_{2}=\frac{1}{2}\left[k_{2}+k_{3}+k_{4}-\sqrt{\left(k_{2}+k_{3}+k_{4}\right)^{2}-4 k_{2} k_{4}}\right]
\end{aligned}
$$

The pre-exponential coefficient terms for the number of molecules in the $\mathrm{W}$ state are given by:

$$
\begin{gathered}
P_{1 W}=\frac{k_{2}+k_{3}-k_{4}+\left(r_{1}-r_{2}\right)}{2\left(r_{1}-r_{2}\right)} \\
P_{2 W}=\frac{-k_{2}-k_{3}+k_{4}+\left(r_{1}-r_{2}\right)}{2\left(r_{1}-r_{2}\right)}
\end{gathered}
$$

while the pre-exponential coefficients for molecules in the $\mathrm{S}$ state are simpler expressions:

$$
\begin{aligned}
& P_{1 S}=-\frac{k_{3}}{\left(r_{1}-r_{2}\right)} \\
& P_{2 S}=\frac{k_{3}}{\left(r_{1}-r_{2}\right)}
\end{aligned}
$$

The sums of the first and second pre-exponential terms for the $\mathrm{W}$ and $\mathrm{S}$ states are equivalent to the pre-exponential terms for the $\mathrm{W}$ state with the exception of a sign change on the $k_{3}$ term in the numerators:

$$
\begin{aligned}
& P_{1 W+S}=\frac{k_{2}-k_{3}-k_{4}+\left(r_{1}-r_{2}\right)}{2\left(r_{1}-r_{2}\right)} \\
& P_{2 W+S}=\frac{-k_{2}+k_{3}+k_{4}+\left(r_{1}-r_{2}\right)}{2\left(r_{1}-r_{2}\right)}
\end{aligned}
$$

Equations S9a, S9b, S12a and S12b are the rate constants and normalized pre-exponential terms for the bi-exponential decay in the molecular population on the surface represented by the histogram plot of the residence times of molecules, given as follows:

$$
H(t)=P_{1 W+S} e^{-r_{1} t}+P_{2 W+S} e^{-r_{2} t}
$$

When the system of these four equations are solved for $k_{2}, k_{3}$, and $k_{4}$ in terms of $P_{1}, P_{2}, r_{1}$, and $r_{2}$, the relationships given in Equations 5-7 in the main body of this article are found which allow the interpretation of single-molecule residencetime histograms in terms of the microscopic rates of desorption, intercalation, and emergence. It is interesting to note that the conditions of this special case do not require the surface to be at equilibrium with the solution at any time during the course of the experiment as long as the free solution concentration, F, remains sufficiently low that the adsorption rate can be neglected.

\section{References.}

(1) Lowry, T. M.; John, W. T. Journal of the Chemical Society, Transactions 1910, 97, 2634.

(2) Wei, J.; Prater, C. D. In Advances in Catalysis; Eley, D. D., Selwood, P. W., Weisz, P. B., Eds.; Academic Press: New York, NY, 1962; Vol. Volume 13, p 203.

(3) Capellos, C. Kinetic systems: mathematical description of chemical kinetics in solution; New York : Wiley-Interscience: New York, 1972. 


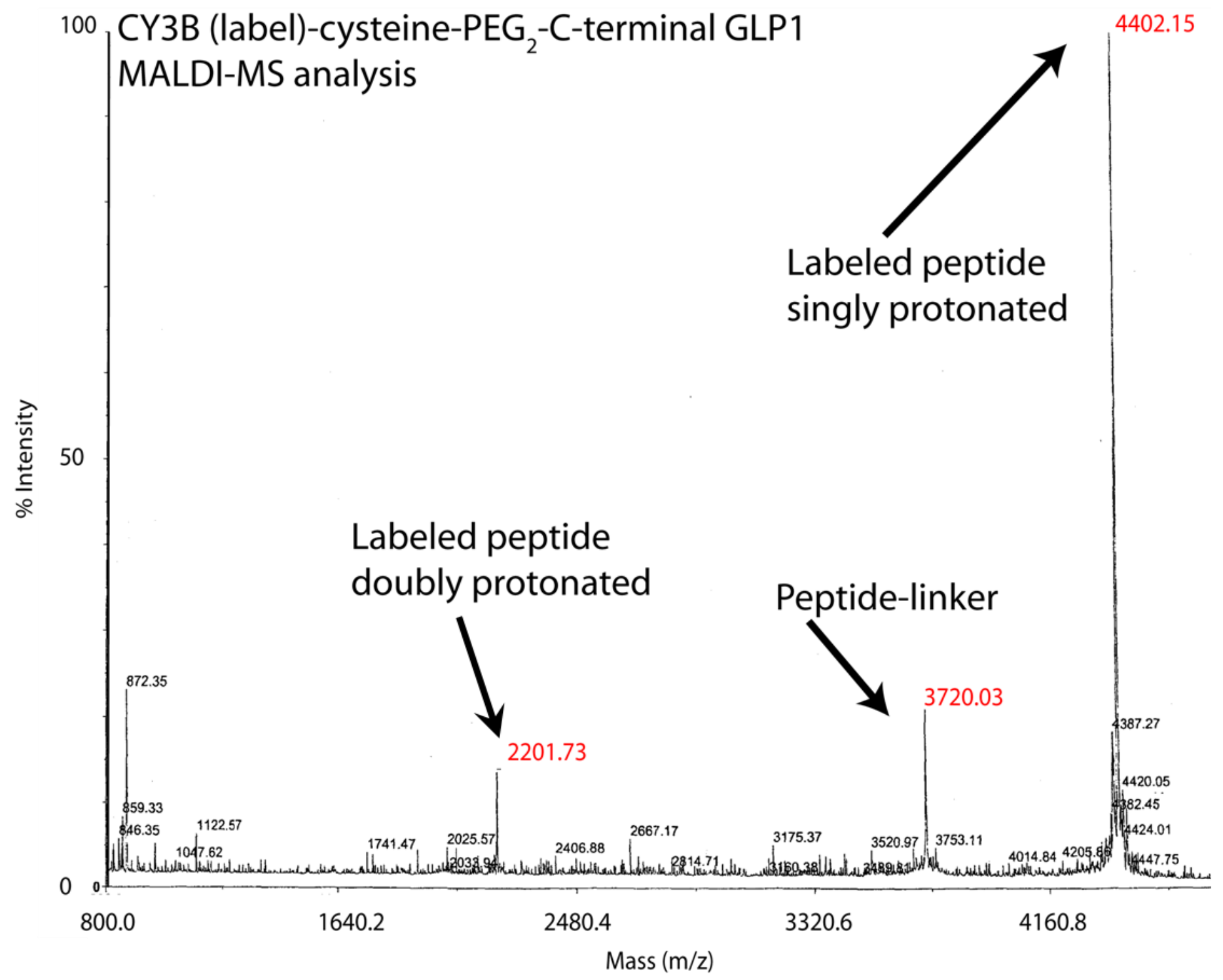

Figure S-1. MALDI analysis of Cy3B-labeled GLP1. The HPLC-purified product was analyzed with time-of-flight matrix-assisted laser desorption ionization time-of-flight mass spectrometry (MALDITOF) in positive ion mode. The labeled peptide consists of the 30-residues of GLP-1 (HAEGTFTSDVSSYLEGQAAKEFIAWLVKGR), connected at the C-terminus to a Novabiochem (01-63-0141) bifunctional 20-atom $\mathrm{PEG}_{2}$ spacer connected to a single amide-capped cysteine labeled at the thiol with GE Amersham (PA63131) Cy3B maleimide. At neutral $\mathrm{pH}$ the entire construct is zwitterionic with a total molecular weight of 4401.10. The singly-protonated species observed in positive-ion mode mass spectrometry has a molecular weight of 4402.93 with a calculated exact mass for the most abundant peak of 4402.11. The doubly-protonated species is also detected, which has an exact mass-to-charge ratio of 2201.56. A small amount of unlabeled peptide-linker-cysteine ( $<20 \%$ of total intensity) is also observed at a mass of 3720.03 , however because experiments are run at surface coverages well below saturation $\left(<10^{-6}\right)$, the competition of unlabeled peptide for binding sites on the lipid bilayer is negligible. Peptide concentrations were determined based only on the labeled peptide population; free label was previously removed by HPLC. 


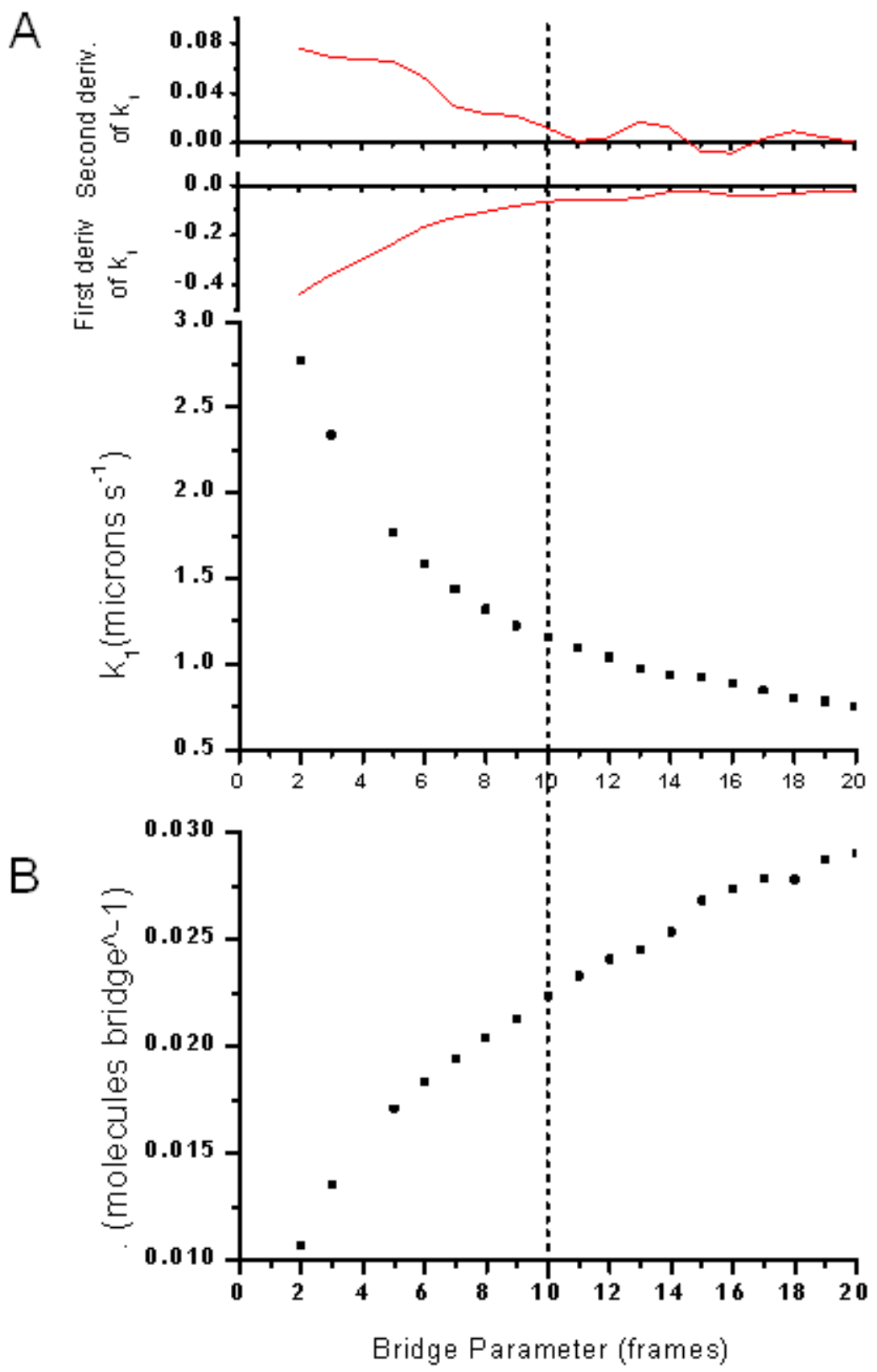

Figure S-2. Optimization of Imaging Algorithm to Bridge Photoblinking Events.

A. Plots of $k_{1}$ and its first and second derivatives, as a function of bridge parameter, where singlemolecule residence-time histograms were constructed for each bridge parameter and the results fit to Equation 1. The value of $k_{1}$ was calculated from Equation 9 using the parameters $r_{1}, r_{2}, P_{1}$, and $P_{2}$ from the histograms. First and second derivatives approach zero when the bridge parameter is increased to ten frames.

B. Plot of the number of molecules estimated to appear in a 9-pixel region from a 50-pM solution at the rate $k_{1}$ from ' $\mathrm{A}$ ' during the bridge time on the $\mathrm{x}$-axis. When the bridge parameter is ten frames, the probability of a second molecule appearing in a 9-pixel region during a bridge of 10 frames or $1.0 \mathrm{sec}$ is $\sim 2.2 \%$. 

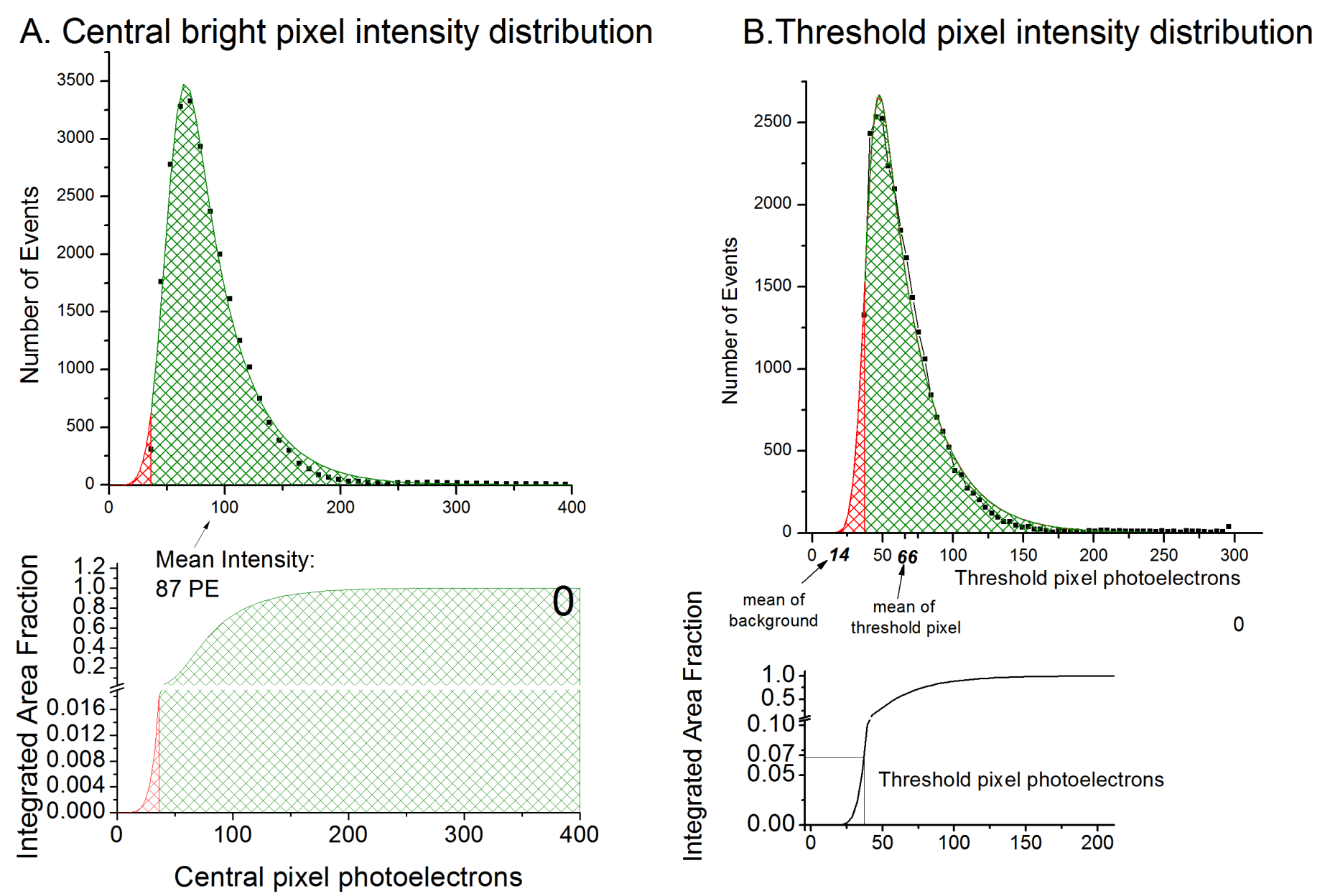

Figure S-3. Intensity Distributions of Cy3B-labeled GLP-1 Fluorescence Spots. A. Distribution of the brightest pixel intensity of labeled-GLP1 fluorescence spots. The mean intensity is 87 photoelectrons (PEs). By fitting the distribution to a Gaussian convoluted with a single-sided exponential, the probability of an event being below the 37-PE critical level (3.0-standard deviations above background) is estimated to be $1.8 \%$. B. Distribution of the next-brightest or threshold pixel intensity of labeledGLP1 fluorescence spots. The mean intensity of this next-brightest pixel distribution is 66 PEs, and the probability of an event being below the 37-PE critical level is $6.9 \%$. Note that integrated area fractions have a y-axis scale change at the line break. 


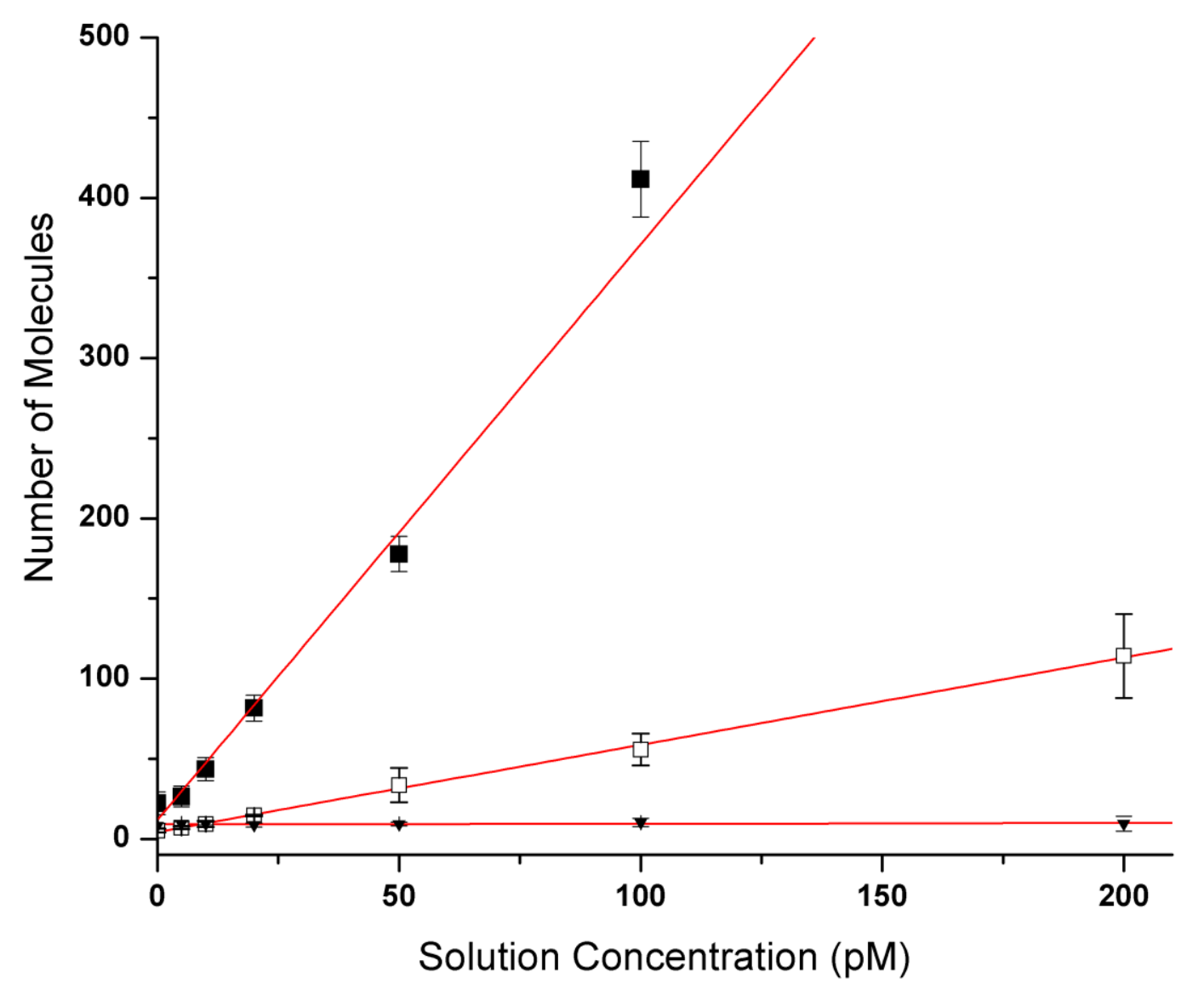

Figure S-4. Isotherms Comparing the Binding of Cy3B-labeled GLP-1 to DPPC with Control Samples. Top line (solid squares) is the binding of Cy3B-labeled GLP-1 to a DPPC bilayer; slope of the fitted line is $3.6( \pm 0.2)$ molecules $\mathrm{pM}^{-1}$. Middle line (open squares) is adsorption of Cy3B-labeled GLP-1 to bare glass, with a fitted line slope of $0.54( \pm 0.03)$ molecules $\mathrm{pM}^{-1}$. Bottom line (solid triangles) is Cy3Blabeled cysteine with a 20-atom polyethylene-glycol linker binding to a DPPC bilayer, with a fitted slope of $0.0040( \pm 0.0003)$ molecules $\mathrm{pM}^{-1}$. 


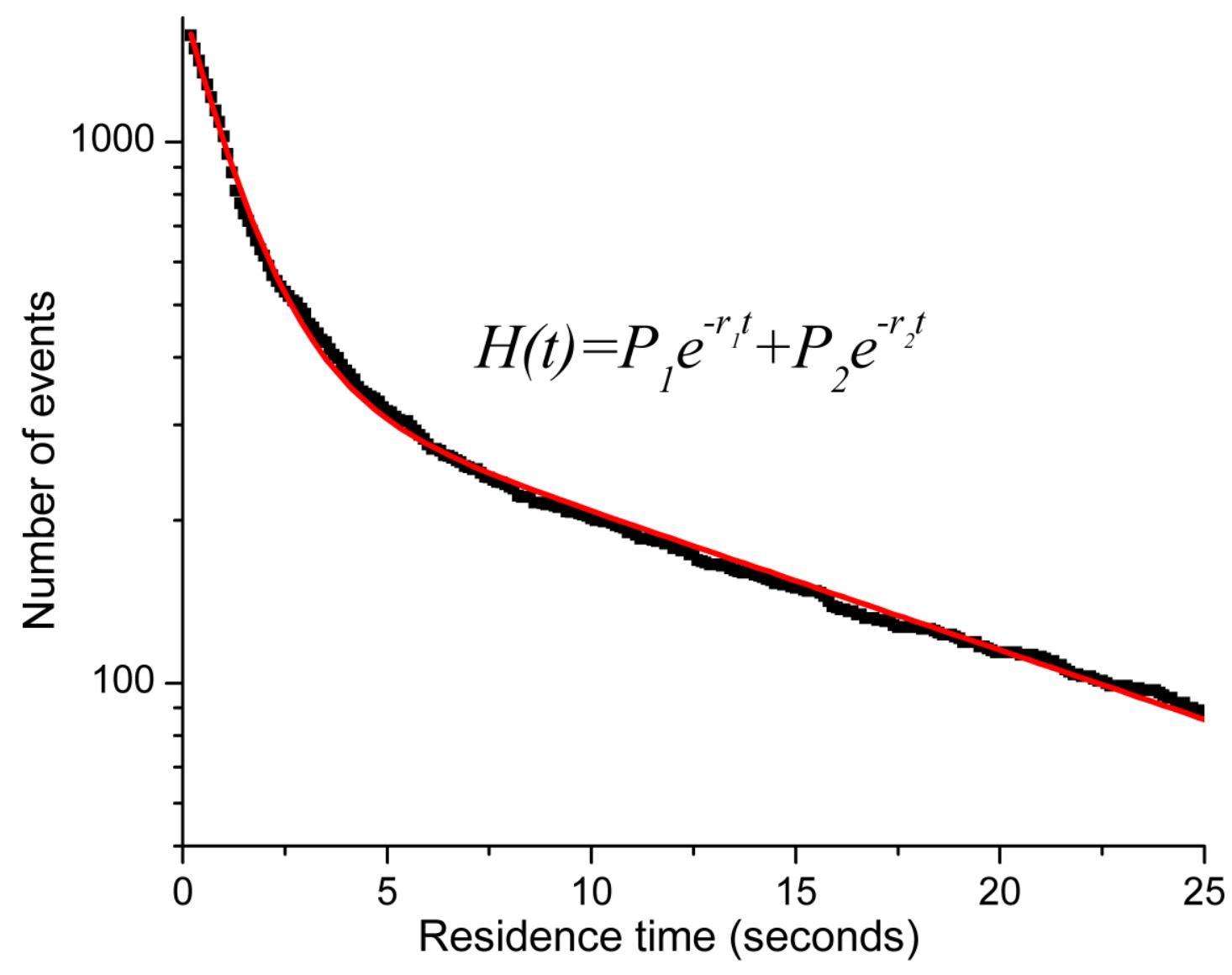

Figure S-5. Example cumulative residence-time histogram for GLP-1 binding to a DPPC supported lipid bilayer, plotted on a log-linear scale. The results clearly show that the data points (black) are not a single-exponential decay but they fit a biexponential model (red line). 


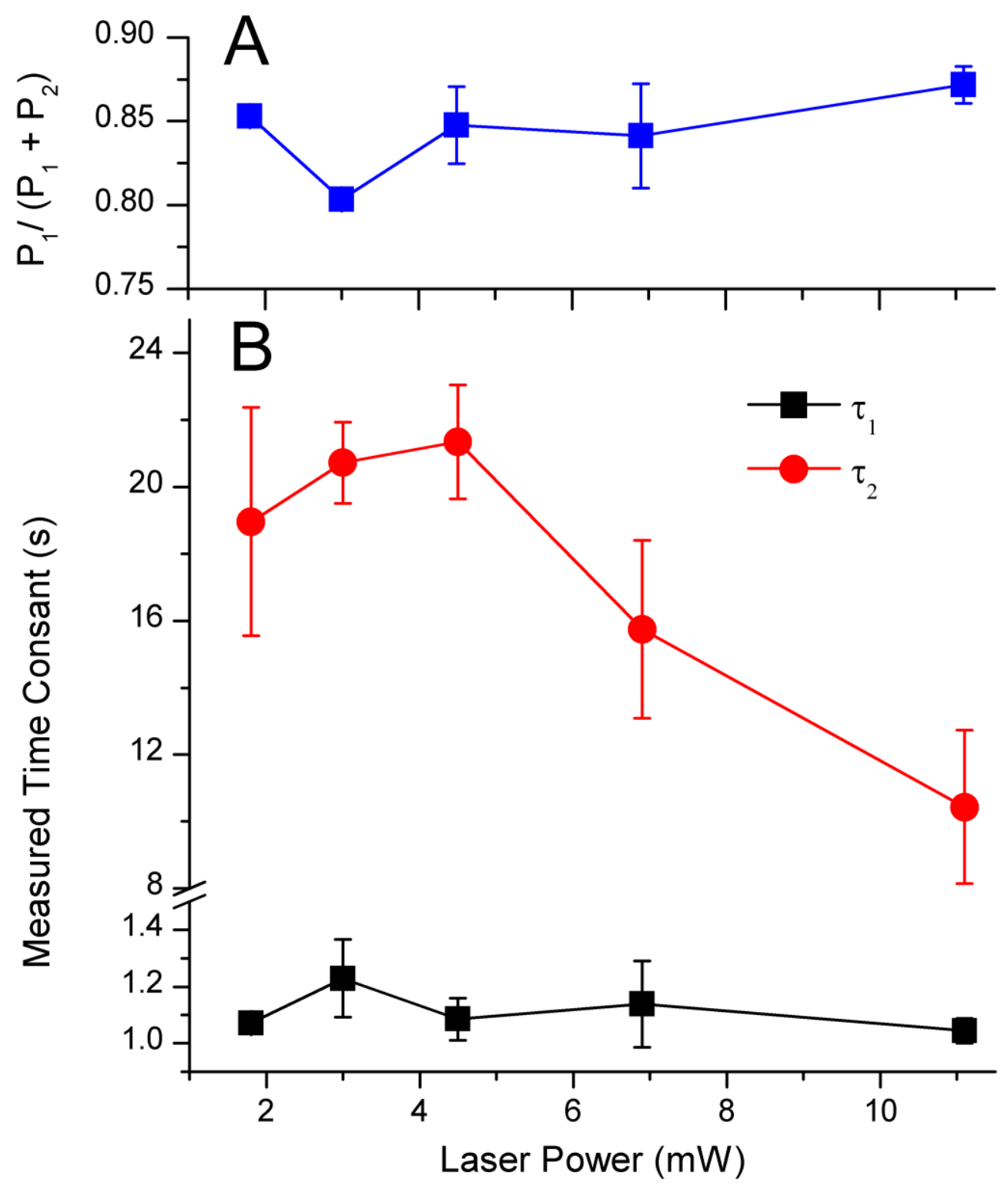

Figure S-6. Residence-time Histogram Kinetics versus Laser Power. A. Pre-exponential fraction, $P_{1} /\left(P_{1}+P_{2}\right)$. B. Fast and slow decay constants, $\tau_{1}$ and $\tau_{2}$, respectively. Note the y-axis break between 1.5 and 8 seconds and the change in scale. The pre-exponential fraction and fast time constant show no correlation with laser power, as does the slow time constant below a laser power of $4.5 \mathrm{~mW}$. Above $4.5 \mathrm{~mW}$, however, the measured slower time constant decreases, suggesting that photobleaching of the dye label shortens the apparent residence times of the longer-lived population. Kinetic parameters reported in the manuscript are for data acquired at a laser power of $3.0 \mathrm{~mW}$, corresponding to an excitation power density of $24 \mathrm{Wcm}^{-2}$ and the sample and well below the level where laser power influences the results. 
Table S-1. Comparison of GLP-1 Kinetic Parameters Measured on Different Systems

\begin{tabular}{|c|c|c|c|c|c|}
\hline $\begin{array}{c}\text { Microscope } \\
\text { System }^{A}\end{array}$ & $\begin{array}{c}\tau_{1} \\
\text { (seconds) }\end{array}$ & $\begin{array}{c}\tau_{2} \\
\text { (seconds) }\end{array}$ & $P_{1} /\left(P_{1}+P_{2}\right)$ & $\begin{array}{c}K_{0} \\
(\mu \mathrm{m})^{\mathrm{B}}\end{array}$ & $\begin{array}{l}\text { Number of events } \\
\text { per experiment / } \\
\text { \# of Experiments }\end{array}$ \\
\hline $\begin{array}{c}\text { Olympus IX71 } \\
514.5-n m \\
\text { Excitation } \\
60 x \text { objective } \\
\text { Cy3 label } \\
\end{array}$ & $1.26 \pm 0.06$ & $20 \pm 3$ & $0.867 \pm 0.005$ & $5.3 \pm 0.1$ & $3500 \pm 400 / 3$ \\
\hline \multirow{2}{*}{$\begin{array}{l}\text { Nikon TE200 } \\
514.5-\mathrm{nm} \\
\text { Excitation } \\
\text { 100x objective } \\
\text { Cy3B label }\end{array}$} & $1.2 \pm 0.1$ & $18.2 \pm 0.9$ & $0.84 \pm 0.01$ & $4.4 \pm 0.2$ & $2400 \pm 700 / 3$ \\
\hline & $1.2 \pm 0.1$ & $21 \pm 2$ & $0.88 \pm 0.02$ & & $1900 \pm 200 / 3$ \\
\hline \multirow{2}{*}{$\begin{array}{l}\text { Olympus IX71 } \\
532-n m \\
\text { Excitation } \\
60 x \text { objective } \\
\text { Cy3B label }\end{array}$} & $1.19 \pm 0.05$ & $19 \pm 2$ & $0.83 \pm 0.03$ & $3.6 \pm 0.2$ & $1200 \pm 300 / 3$ \\
\hline & $1.2 \pm 0.1$ & $21 \pm 1$ & $0.8033 \pm 0.0005$ & & $2700 \pm 500 / 2$ \\
\hline
\end{tabular}

${ }^{\mathrm{A}}$ Microscope frame, laser-excitation wavelength, TIRF-objective power, and dye label used on GLP-1.

${ }^{\text {B }}$ For missing entries, residence-time histograms at 50-pM GLP-1 were replicated (on different days) but no isotherm data were collected. 